\title{
Multivariable model development and internal validation for prostate cancer specific survival and overall survival after whole-gland salvage lodine-125 prostate brachytherapy
}

Max Peters ${ }^{1}$, MD; Jochem R.N. van der Voort van Zyp ${ }^{1}$, MD, PhD; Marinus A. Moerland ${ }^{1}$, PhD; Carel J. Hoekstra ${ }^{2}$, MD; Sandrine van de Pol ${ }^{2}$, MD; Hendrik Westendorp ${ }^{2}, \mathrm{MSc}$; Metha Maenhout ${ }^{1}$, MD; Rob Kattevilder ${ }^{2}$, BSc; Helena M. Verkooijen ${ }^{3}$, MD, PhD; Peter S.N. van Rossum ${ }^{1}$, MD; Hashim U. Ahmed ${ }^{4,5}$, MD, PhD, FRCS (Urology); Taimur T. Shah ${ }^{4-6}$, MD; Mark Emberton ${ }^{4,5,7}$, BSc, MBBS, MD, FRCS (Urology); Marco van Vulpen ${ }^{1}, \mathrm{MD}, \mathrm{PhD}$.

1. Department of Radiation Oncology, University Medical Center Utrecht, The Netherlands.

2. Radiotherapeutic Institute RISO, Deventer, The Netherlands.

3. Imaging division, University Medical Center Utrecht, The Netherlands.

4. Division of Surgery and Interventional Science, University College London, UK;

5. Department of Urology, UCLH NHS Foundation Trust, UK.

6. Department of Urology, Whittington Hospital NHS Trust, London, UK

7. NIHR UCLH/UCL Comprehensive Biomedical Research Centre, London, UK

Correspondence:

University Medical Center Utrecht

Department of Radiotherapy, HP. Q00.118

Heidelberglaan 100, 3584CX Utrecht, The Netherlands

Phone: 0031-88-7558800

Email: M.Peters-10@umcutrecht.nl

Key words: whole-gland salvage, prostate cancer, 1125 brachytherapy, predictive factors, survival.

Running title: A multivariable model for prostate cancer specific and overall survival after salvage lodine125 prostate brachytherapy

Word count: 2717 (including abstract, manuscript and figure legends)

Conflicts of interest and funding:

M. Emberton and H.U. Ahmed would like to acknowledge funding from the Medical Research Council (UK), the Pelican Cancer Foundation Charity, Prostate Cancer UK, St Peters Trust Charity, Prostate Cancer Research Centre the Wellcome Trust, National Institute of Health Research-Health Technology Assessment Programme, and the US National Institute of Health-National Cancer Institute. M. Emberton receives funding in part from the UK National Institute of Health Research UCLH/UCL Comprehensive Biomedical Research Centre. M. Emberton and H.U. Ahmed receive funding from USHIFU, GSK and Advanced Medical Diagnostics for clinical trials. M. Emberton is a paid consultant to Steba Biotech and USHIFU. Both have previously received consultancy payments from Oncura/GE Healthcare and Steba Biotech. 


\begin{abstract}
Background: Whole-gland salvage lodine-125-brachytherapy is a potentially curative treatment strategy for localised prostate cancer (PCa) recurrences after radiotherapy. Prognostic factors influencing PCaspecific and overall survival (PCaSS \& OS) are not known. The objective of this study was to develop a multivariable, internally validated prognostic model for survival after whole-gland salvage I-125brachytherapy.

Materials and methods: Whole-gland salvage I-125-brachytherapy patients treated in the Netherlands from 1993-2010 were included. Eligible patients had a transrectal ultrasound-guided biopsy-confirmed localised recurrence after biochemical failure (clinical judgement, ASTRO or Phoenix-definition). Recurrences were assessed clinically and with $\mathrm{CT}$ and/or MRI. Metastases were excluded using CT/MRI and technetium-99m scintigraphy. Multivariable Cox-regression was used to assess the predictive value of clinical characteristics in relation to PCa-specific and overall mortality. PCa-specific mortality was defined as patients dying with distant metastases present. Missing data was handled using multiple imputation (20 imputed sets). Internal validation was performed and the C-statistic calculated. Calibration plots were created to visually assess the goodness-of-fit of the final model. Optimismcorrected survival proportions were calculated. All analyses were performed according to the TRIPOD statement.

Results: Median total follow-up was 78 months (range 5-139). A total of 62 patients were treated, of which $28(45 \%)$ died from PCa after mean ( \pm SD) $82( \pm 36)$ months. Overall, 36 patients (58\%) patients died after mean $84( \pm 40)$ months. PSA doubling time (PSADT) remained a predictive factor for both types of mortality (PCa-specific and overall): corrected hazard ratio's (HR's) 0.92 (95\%-Cl: 0.86-0.98, p=0.02) and 0.94 (95\%-Cl: 0.90-0.99, $\mathrm{p}=0.01$ ), respectively (C-statistics 0.71 and 0.69 , respectively). Calibration was accurate up to 96 months follow-up. Over $80 \%$ of patients can survive 8 years if PSADT>24 months (PCaSS) and $>33$ months (OS). Only approximately $50 \%$ survival is achieved with a PSADT of 12 months. Conclusion: A PSADT of respectively $>24$ months and $>33$ months can result in $>80 \%$ probability of PCaspecific and overall survival 8 years after whole-gland salvage l-125-brachytherapy. Survival should be weighed against toxicity from a salvage procedure. Larger series and external validation are necessary.
\end{abstract}




\section{Introduction}

Brachytherapy and external beam radiotherapy (EBRT) can achieve high tumour control in patients with primary prostate cancer ${ }^{1,2}$. EBRT dose escalation has led to further improvement of these results ${ }^{1,3}$. However, depending on tumour characteristics, PSA-parameters and use of androgen deprivation therapy (ADT), biochemical recurrences can still occur in $30-50 \%$ of patients after $5-10$-years ${ }^{1,3}$. Over $80 \%$ of patients can harbour a prostate-only recurrence ${ }^{4}$. Local salvage therapy is a potentially curative treatment for these prostate-confined recurrences. Whole-gland salvage can be performed using different techniques and is able to achieve long term biochemical control and survival and thereby postpone the use of androgen deprivation therapy (ADT) in carefully selected patient groups $s^{5-9}$. Risk factors for failure and mortality for salvage brachytherapy have not been comprehensively defined, because of small series with often limited events ${ }^{5,9,10}$. A few series have defined pre-salvage PSA, PSAdoubling time (PSADT) and time-to-relapse after primary therapy as possible predictors of biochemical failure after salvage brachytherapy ${ }^{11-13}$. Although biochemical failure often precedes the development of distant metastases and death ${ }^{1}$, no predictive factors for survival have as of yet been identified. In this report, we therefore aimed to develop a prognostic model for PCa-specific survival (PCaSS) and overall survival (OS) after whole-gland salvage 125-brachytherapy, based on the largest salvage I-125brachytherapy cohort. 


\section{Materials and Methods}

Patient selection, data collection and outcome assessment

The institutional review board of the University Medical Center Utrecht (UMCU) permitted the analysis of the data. In total, 62 patients were treated with whole-gland salvage l-125-brachytherapy from November 1993 until April 2010 in both the University Medical Center, Utrecht $(n=33)$ and the Radiotherapeutic institute RISO, Deventer, the Netherlands ( $n_{-29}$ ). Selection was based on objective assessment of a localised recurrence after biochemical failure (according to clinical judgement and the ASTRO or Phoenix definition after 1996 and 2005, respectively). Localised disease was transrectal ultrasound-guided biopsy-confirmed, with absence of lymph node or distant metastases based on pelvic CT and/or MRI ( $n=22$ ) and technetium-99m scintigraphy (bone-scan). Transrectal ultrasound (in all patients) or MRI (in 22 patients) was used to exclude capsular extension. PSA, PSA-kinetics and other prognostic factors before primary therapy and salvage were not used for selection and were judged by the treating radiation oncologist. ADT was discontinued at salvage.

Survival data was obtained by the primary researcher (MP) from patient charts. Survival was subdivided in prostate cancer specific survival (PCaSS) and overall survival (OS) after salvage brachytherapy. PCaSS was separately evaluated using the records by two independent radiation oncologists (JVZ, CH). For death due to prostate cancer, it was necessary to have distant metastases.

\section{Toxicity}

Late ( $>6$ months post-implantation) severe ( $\geq$ grade 3 ) gastrointestinal (GI) and genitourinary (GU) toxicity was evaluated with the common terminology criteria for adverse events (CTCAE 4.03).

\section{Implantation details}

The prescription for the prostatic V100 (volume of the prostate receiving $100 \%$ dose [ $=145 \mathrm{~Gy}]$ ) was $\geq 95 \%$. The D90 (minimal dose received by $90 \%$ of the prostate) was $\geq 145 \mathrm{~Gy}$. In the UMCU images were imported in the Sonographic Planning of Oncology Treatment planning software (SPOT, Nucletron BV, Veenendaal, the Netherlands). The planning system in the RISO consisted of subsequent versions of Variseed $^{\mathrm{TM}}$ (Varian Medical Systems, Palo Alto, CA). Loose and stranded seeds were both used in this cohort.

\section{Analysed predictive factors}

Factors before primary radiotherapy consisted of primary treatment (lodine-125 brachytherapy or EBRT/IMRT), dose (>64.4 Gy or $\leq 64.4 \mathrm{~Gy}$ ), initial PSA (iPSA), T stage $(1,2$ or 3 ) and initial Gleason grade (2-6, 7, or 8-10). Sub-classification of T-stage was frequently missing, so only the overall T-stage was used. Factors before salvage brachytherapy were PSA-nadir (i.e. the lowest PSA-value) after primary treatment, (biochemical) disease free survival interval (DFSI) after primary radiotherapy, age, PSA, PSADT, PSA-density (=PSA-value divided by the prostatic volume as assessed on ultrasound), PSAvelocity and ADT-use. Gleason score pre-salvage was not analysed because of the chance of misclassification due to radiation effects (and therefore frequently missing scores) ${ }^{14}$. PSA kinetics (PSA- 
velocity and PSADT) were calculated using the Memorial Sloan Kettering Cancer Center calculator ${ }^{15}$. PSA-nadir after salvage was separately analysed, because of its redundancy in patient selection. No interactions between variables were considered.

\section{Statistical analysis}

Normally distributed variables are presented as mean $( \pm S D)$, skewed distributed variables as medians with ranges, and categorical data as frequencies with percentages. Kaplan-Meier survival analyses for PCaSS and OS were performed for categories of possible predictor variables. Categories of predictors were based on common prognostic categories from the literature (e.g. PSA $\leq 10$ and $>10 \mathrm{ng} / \mathrm{ml}$ and PSADT $\leq 10$ months and $>10$ months $^{16}$ ). Receiver operating characteristic (ROC)-analysis was performed for PSA-density and PSA-velocity to identify cutoff values with maximal sensitivity and specificity, since groups were unequal when categories based on the literature were adopted. Differences were tested with the log-rank test.

Missing data was considered at random. Multiple imputation (MI) with the iterative Markov Chain Monte Carlo method was performed (20 iterations) $)^{17,18}$. The MI-procedure was performed with all predictors listed above in the imputation process, including the outcome (PCaSS and OS) ${ }^{18,19}$.

\section{Model building}

Univariable and multivariable Cox-proportional hazards regression was performed. Before Coxregression, correlation coefficients between PSA, PSA kinetic factors and other predictors were calculated. Pearson's correlation coefficient was used in case of linear relations and Spearman's rank correlation in case of a non-linear correlation. Factors were excluded from multivariable analysis when collinearity was present (i.e. correlation coefficient $\geq 0.75$ ). When (multi)collinearity was present, the factor measurable with the least practical effort was given priority to aid clinical application. From univariable analysis, factors were selected for multivariable analysis if $p<0.10$ based on the Wald-test. Stepwise backward elimination was used for the multivariable analysis and the models were compared at each step with the likelihood ratio test statistic. Proportional hazards was visually evaluated with loglog curves for categorical predictors and Schoenfeld residuals for continuous variables. Survival

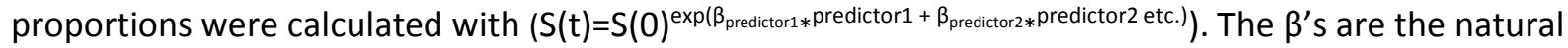
logarithm of the hazard ratio's from multivariable analysis. $S(0)$ is the baseline survival proportion at a specified follow-up point with determinants from multivariable analysis equaling 0 . No correction for multiple testing was performed since parameters were often highly correlated with one another, thereby possibly inducing type II errors when conservatively adjusting the $\alpha$-level.

\section{Internal validation and calibration}

Harrell's C-statistic (concordance index) was used as a discrimination measure, comparable to an areaunder-the-curve (AUC) value from logistic regression ${ }^{20}$. With 500 bootstrapping resamples for each of the 20 imputed datasets, the optimism of the model and shrinkage factor for the coefficients were calculated, after which the C-statistic and coefficients (and subsequently HR's) could be adjusted. 
The predictive accuracy for PCaSS and OS was visualised with calibration plots at 5 and 8-years. No external validation could be performed. Kaplan-Meier survival analysis, MI and Cox-proportional hazards regression procedures were performed using IBM SPSS version 20 (statistical package for the social sciences Inc, Chicago, IL). $\mathrm{R}$ language environment (version 3.1.2) for statistical computing (available at http://www.r-project.org/ ${ }^{21}$ ) was used for calibration and internal validation (survival and rms package). Statistical significance was set at $p \leq 0.05$. All procedures and reporting were based on the transparent reporting of multivariable prediction models for individual prognosis or diagnosis (TRIPOD) statement ${ }^{22}$.

\section{Results}

\section{Patient characteristics}

Primary radiotherapy consisted of $64.4 \mathrm{~Gy}$ in 23 fractions of $2.3 \mathrm{~Gy}$ in the majority of patients ( $\mathrm{n}=26$, $45 \%)$. Most patients had favourable tumor characteristics before primary radiotherapy and salvage, although outliers were present (e.g. PSA before salvage was $92.6 \mathrm{ng} / \mathrm{ml}$ in one patient, with the second highest $25.5 \mathrm{ng} / \mathrm{ml}$ ) (Table 1). Response to salvage was present in 51 (82\%) of 62 patients. Median total follow-up was 78 months (range 5-139). In total, 36 patients (58\%) patients died during follow-up. The mean time to death was $84( \pm 40)$ months. Twenty-eight $(45 \%)$ patients died due to PCa after $82( \pm 36)$ months. Eight patients died unrelated to the PCa, six of whom did not experience biochemical failure.

\section{Toxicity}

Data to assess $\mathrm{GI}$ and GU toxicity was available for 60 and 61 patients, respectively. A total of 12 patients $(20 \%)$ experienced radiation proctitis for which they were treated with argon plasma laser coagulation. Late $\geq$ grade $3 \mathrm{GU}$ toxicity was present in 18 patients $(30 \%)$. Urethral strictures $(n=10)$ and urinary retention $(n=4)$ were most frequently observed. Finally, 5 patients $(8 \%)$ were observed with a combined toxicity profile with both $\mathrm{GI}$ and GU toxicity: two patients had a grade 3 and one patient a grade 4 rectovesical fistula. Lastly, two patients experienced a grade 3 rectourethral fistula.

\section{Kaplan-Meier survival analysis}

The 10 -year estimated PCaSS was $43 \%$, with median survival of 108 months ( $95 \% \mathrm{Cl}$ : $91-125)$ ). After $10-$ years, OS was $34 \%$, with a median survival of 104 months (95\% Cl: $94-115)$.

Patients with a pre-salvage PSA $\leq 10 \mathrm{ng} / \mathrm{ml}$ had 10 -year PCaSS of $65 \%$ versus $15 \%$ for patients with PSA $>10 \mathrm{ng} / \mathrm{ml}$ (log rank: $p<0.0001$ ). OS also differed, with 10 -year OS being $53 \%$ versus $12 \%$, respectively $(p<0.0001)$. Patients with a PSADT $>10$ months compared to $\leq 10$ months had a 10 -year PCaSS of $71 \%$ versus $22 \%$, $(p=0.002)$, and an OS of $47 \%$ versus $21 \%(p=0.03)$, respectively. Other prognostic variables significantly associated with decreased PCaSS and OS were DFSI $<36$ months, PSA-density $>0.25 \mathrm{ng} / \mathrm{ml} / \mathrm{cc}$, PSA-velocity $>3 \mathrm{ng} / \mathrm{ml} /$ year and nadir after salvage $>1.0 \mathrm{ng} / \mathrm{ml}$. Primary patient and tumor characteristics were not associated with survival after salvage. Primary Gleason 8-10 tumours were almost significantly related to survival compared to Gleason 2-6 and 7, but the group consisted of only 3 cases. KaplanMeier results are depicted in Table 2 and supplementary figures. 


\section{Missing data}

No outcome data was missing. Predictor variables not standardised in follow-up had the most missing values (PSA-velocity and DFSI, both 11 [17.7\%]). There was significant overlap in missing data, with approximately $80 \%$ of cases having no missing values. Because of no intrinsic relation with the missing values or the outcomes, data was considered missing at random. Table 1 lists further information on missing data.

\section{Correlation}

Pre-salvage PSA and PSA-density showed the highest correlation (Pearson correlation coefficient 0.95, $\mathrm{p}<10^{-29}$ ). Pre-salvage PSA and PSA-velocity were also highly correlated (Spearman's rank correlation 0.8, $p<10^{-11}$ ). All other significant correlations were $<0.75$. The largest of these correlation was between presalvage PSA and DFSI (Spearman: $-0.65, p<10^{-6}$ ), DFSI and PSADT (Pearson: 0.58, $p<0.0001$ ) and between PSA and nadir-after salvage (Spearman: 0.51, $p<0.001$ ). Due to these correlations, PSA-density and PSAvelocity were excluded from the multivariable Cox-regression analysis. PSA was kept in, because it consists of only a single measurement.

\section{Modelling results, internal validation and calibration}

Univariable analysis showed PSA, PSADT, PSA-velocity and PSA-nadir after salvage as significant predictors for PCaSS and OS. For PCaSS, PSA-density and DFSI were almost significant $(p=0.06$ and $p=0.054)$. For OS, significance of DFSI disappeared $(p=0.13)$. Univariable, ADT use was almost significant for OS ( $p=0.08$ ). After multivariable analysis for PCaSS (variables included: DFSI, PSA and PSADT), only PSADT remained as a significant predictor: corrected hazard ratio (HR) 0.92 (95\%-Cl: 0.86-0.98, $p=0.02$ ), indicating an approximate $8 \%$ decrease in hazard for PCaSS for each month increase in PSADT. PSADT remained a significant predictor for OS after multivariable analysis (variables included: ADT, PSA and PSADT): corrected HR 0.94 (95\%-Cl: 0.90-0.99, $p=0.01$ ), respectively (Table 3). There were no violations of the proportional hazards assumption. The apparent C-statistic was 0.73 for PCaSS 0.71 for OS and adjusted 0.71 and 0.69 , respectively. Shrinkage factors were 0.77 for PCaSS and 0.71 for OS. Calibration plots after 5 and 8 years are depicted in Figure 1. Observed survival frequencies are concordant with predicted probabilities from the final models up to 8 years.

\section{Survival proportions}

Baseline cumulative survival proportions $(\mathrm{S}(0))$ for PCaSS and OS at 8 years were 0.215 and 0.219 , respectively. In patients with a pre-salvage PSADT>24 months, whole-gland salvage l-125-brachytherapy results in a $>80 \%$ chance of PCaSS up to 8 years. For OS, a PSADT $>33$ months results in this survival percentage (Figure 2 and supplementary table 4). With a PSADT of 12 months or lower, survival dropped to approximately $\leq 50 \%$.

\section{Discussion}

In summary, our study has shown a relation with several pre-salvage characteristics and both prostate cancer specific and overall survival after whole-gland salvage I-125-brachytherapy for localised prostate 
cancer recurrences after primary radiotherapy. After multivariable Cox analysis, only PSADT remained a predictor of both PCaSS and OS: corrected hazard ratio (HR) 0.92 (95\%-Cl: 0.86-0.98, p=0.02) and 0.94 (95\%-Cl: $0.90-0.99, p=0.01)$, respectively. This implies a decrease in hazard for mortality of approximately $8 \%$ and $6 \%$ with every month increase in PSADT, respectively.

To our knowledge these 62 patients constitute the largest whole-gland salvage l-125-brachytherapy series, with sufficient events for prognostic modelling. We have used the recent TRIPOD statement regarding the conduct and reporting of prognostic research ${ }^{22}$, to ensure transparent reporting of handling variables, missing data, model building, validation and calibration. We trust this might facilitate comparison with future groups reporting on salvage I-125-brachytherapy outcomes. For now, with the optimism-corrected hazard ratio's for PSADT and baseline survival proportion, exact survival percentages can be calculated in clinical practice for individual patients.

Nonetheless, our study has some limitations. At 8 years, calibration was decent for both PCaSS and OS, although with a relatively large spread as is visible in the wide confidence intervals of the observed (Kaplan-Meier) survival estimates. The same is applicable to the predicted survival probabilities. Larger groups and possibly other predictors are necessary to add precision to the current estimates.

It is possible that factors not taken into account for this analysis, such as pre-salvage Gleason grade ${ }^{8}$ and morphological findings on MRI (and/or functional sequences), might have additional predictive ability for progression and survival. These were not available in the current patient cohort. Furthermore, a type I error in one/some of the assessed parameters is a possibility due to multiple testing. However, parameters were often (highly) correlated with one another, thereby reducing the risk of a type I error. Also, correcting the $\alpha$-level conservatively with a Bonferroni, Šídák or Holm's Sequential Bonferroni procedure would assume independence of the separate tests and therefore automatically increase the chance of a type II error (i.e. incorrect non-significant results). Furthermore, PSADT had a significant relation with both types of mortality, making a type I error less likely (even though both types of mortality show overlap). Lastly, an explorative approach was adopted for this set of clinical variables, since this database is the largest nowadays for whole-gland salvage I-125-brachytherapy. This could provide valuable insight into which variables are important for future research into the topic. They do have to be verified in other series, however.

Interestingly, factors before primary radiotherapy do not show an effect on survival. Selection of salvage patients is often performed based on these characteristics ${ }^{16,23}$. These findings could be an indication of their relative insignificance for patient selection. Contrary to this hypothesis, Gleason 8-10 before primary radiotherapy consisted of only 3 patients and showed a tendency in the Kaplan-Meier analysis to low survival. The prognostic relevance of this factor could have been underestimated in our analysis. Quantification can be provided when larger numbers are assessable.

Lastly, without external validation this model cannot provide clinically robust estimates of predicted survival. External validation could decrease the predictive accuracy of PSADT as found in this population, since the current cohort consisted of a relatively dated and high risk population. This uncertainty needs to be taken into account when selecting whole-gland salvage I-125-brachytherapy patients based on their PSADT. However, as this is the only predictive factor from extensive multivariable analysis in predicting survival after whole-gland salvage prostate l-125-brachytherapy so far, it could provide additional guidance in the selection of patients in clinical practice or to intensify post-salvage follow-up 
in patients deemed at high risk for treatment failure and mortality. Patients with a PSADT $<20$ months require at least additional follow-up after salvage to assess disease progression or salvage should be offered earlier or perhaps not at all. Furthermore, the benefits of treatment regarding cancer control/survival need to be weighed against the exacerbated toxicity after whole-gland salvage I-125brachytherapy, as was observed in this and previous series ${ }^{5,6}$.

Other series have provided risk factors for biochemical failure with small groups of whole-gland salvage LDR I-125/Pd-103 and HDR Ir-192-brachytherapy patients and even focal salvage approaches (directed locally at the recurrence) $)^{10-13,24-30}$. Only a few of the whole-gland salvage brachytherapy studies provide multivariable modelling, with often limited events, categorisation of predictor variables and insufficient description of handling missing data, leading to imprecise estimates ${ }^{11-13,26}$. However, there is no data on predictive factors for survival (PCaSS and OS). Predictive factors for survival in other salvage modalities (such as radical prostatectomy and cryosurgery) are more comprehensively defined, because of larger patient numbers and longer follow-up ${ }^{7,8}$. These factors (PSA pre-salvage, primary Gleason score, T-stage [both only univariable] and nadir after salvage, among other) are possibly important for salvage brachytherapy as well, but are not quantified in this setting with multivariable models. In addition, although biochemical failure often precedes the development of distant metastases and (PCa-specific) mortality ${ }^{1}$, it is unclear whether predictive factors associated with biochemical failure can be extrapolated to mortality in the case of salvage brachytherapy patients. Due to often more advanced age at salvage, it is questionable whether these factors will also predict survival in the same manner. Therefore, this analysis has provided the first identification of a pre-savage characteristic which can predict both types of survival in whole-gland salvage I-125-brachytherapy. The PSADT could be used to select patients or identify patient with a poor response to salvage and subsequently intensify follow-up or reject further salvage. The toxicity from such a procedure should be taken into account with this consideration as well.

\section{Conclusion}

PSADT is the only predictive factor for survival after whole-gland salvage I-125-brachytherapy. More than $80 \%$ survival can be achieved with PSADT>24 months (PCaSS) and $>33$ months (OS) up to 8 years after salvage. Only approximately 50\% survival is achieved with a PSADT of 12 months and therefore, survival should be weighed against potential toxicity from a salvage procedure. Larger series, other predictive factors and external validation are warranted. 


\section{References}

1. Zumsteg ZS, Spratt DE, Romesser PB, et al. The natural history and predictors of outcome following biochemical relapse in the dose escalation era for prostate cancer patients undergoing definitive external beam radiotherapy. Eur Urol. 2014. doi: S0302-2838(14)00965-8 [pii].

2. Peinemann F, Grouven U, Bartel C, et al. Permanent interstitial low-dose-rate brachytherapy for patients with localised prostate cancer: A systematic review of randomised and nonrandomised controlled clinical trials. Eur Urol. 2011;60(5):881-893. doi: 10.1016/j.eururo.2011.06.044 [doi].

3. Zelefsky MJ, Pei X, Chou JF, et al. Dose escalation for prostate cancer radiotherapy: Predictors of longterm biochemical tumor control and distant metastases-free survival outcomes. Eur Urol. 2011;60(6):1133-1139. doi: 10.1016/j.eururo.2011.08.029 [doi].

4. Menard C, lupati D, Publicover J, et al. MR-guided prostate biopsy for planning of focal salvage after radiation therapy. Radiology. 2015;274(1):181-191. doi: 10.1148/radiol.14122681 [doi].

5. Ramey SJ, Marshall DT. Re-irradiation for salvage of prostate cancer failures after primary radiotherapy. World J Urol. 2013;31(6):1339-1345. doi: 10.1007/s00345-012-0953-7 [doi].

6. Parekh A, Graham PL, Nguyen PL. Cancer control and complications of salvage local therapy after failure of radiotherapy for prostate cancer: A systematic review. Semin Radiat Oncol. 2013;23(3):222234. doi: 10.1016/j.semradonc.2013.01.006 [doi].

7. Wenske S, Quarrier S, Katz AE. Salvage cryosurgery of the prostate for failure after primary radiotherapy or cryosurgery: Long-term clinical, functional, and oncologic outcomes in a large cohort at a tertiary referral centre. Eur Urol. 2013;64(1):1-7. doi: 10.1016/j.eururo.2012.07.008 [doi]. 
8. Chade DC, Shariat SF, Cronin AM, et al. Salvage radical prostatectomy for radiation-recurrent prostate cancer: A multi-institutional collaboration. Eur Urol. 2011;60(2):205-210. doi:

10.1016/j.eururo.2011.03.011 [doi].

9. Alongi F, De Bari B, Campostrini F, et al. Salvage therapy of intraprostatic failure after radical externalbeam radiotherapy for prostate cancer: A review. Crit Rev Oncol Hematol. 2013. doi:

10.1016/j.critrevonc.2013.07.009; 10.1016/j.critrevonc.2013.07.009.

10. Chen CP, Weinberg V, Shinohara K, et al. Salvage HDR brachytherapy for recurrent prostate cancer after previous definitive radiation therapy: 5-year outcomes. Int J Radiat Oncol Biol Phys.

2013;86(2):324-329. doi: 10.1016/j.ijrobp.2013.01.027 [doi].

11. Moman MR, van der Poel HG, Battermann JJ, Moerland MA, van Vulpen M. Treatment outcome and toxicity after salvage 125-I implantation for prostate cancer recurrences after primary 125-I implantation and external beam radiotherapy. Brachytherapy. 2010;9(2):119-125. doi: 10.1016/j.brachy.2009.06.007 [doi].

12. Henriquez I, Sancho G, Hervas A, et al. Salvage brachytherapy in prostate local recurrence after radiation therapy: Predicting factors for control and toxicity. Radiat Oncol. 2014;9:102-717X-9-102. doi: 10.1186/1748-717X-9-102 [doi].

13. Grado GL, Collins JM, Kriegshauser JS, et al. Salvage brachytherapy for localized prostate cancer after radiotherapy failure. Urology. 1999;53(1):2-10. doi: S0090-4295(98)00492-0 [pii].

14. Crook J, Malone S, Perry G, Bahadur Y, Robertson S, Abdolell M. Postradiotherapy prostate biopsies: What do they really mean? results for 498 patients. Int J Radiat Oncol Biol Phys. 2000;48(2):355-367. doi: S0360-3016(00)00637-4 [pii]. 
15. Memorial Sloan Kettering Cancer Center. Prostate cancer nomograms: PSA doubling time and PSA velocity; http://Www.mskcc.org/nomograms/prostate/psa-doubling-time. Sep. 2014 - Dec. 2014.

16. Nguyen PL, D'Amico AV, Lee AK, Suh WW. Patient selection, cancer control, and complications after salvage local therapy for postradiation prostate-specific antigen failure: A systematic review of the literature. Cancer. 2007;110(7):1417-1428. doi: 10.1002/cncr.22941 [doi].

17. Janssen KJ, Donders AR, Harrell FE,Jr, et al. Missing covariate data in medical research: To impute is better than to ignore. J Clin Epidemiol. 2010;63(7):721-727. doi: 10.1016/j.jclinepi.2009.12.008 [doi].

18. Sterne JA, White IR, Carlin JB, et al. Multiple imputation for missing data in epidemiological and clinical research: Potential and pitfalls. BMJ. 2009;338:b2393. doi: 10.1136/bmj.b2393 [doi].

19. Moons KG, Donders RA, Stijnen T, Harrell FE,Jr. Using the outcome for imputation of missing predictor values was preferred. J Clin Epidemiol. 2006;59(10):1092-1101. doi: S0895-4356(06)00060-6 [pii].

20. Harrell FE,Jr, Lee KL, Mark DB. Multivariable prognostic models: Issues in developing models, evaluating assumptions and adequacy, and measuring and reducing errors. Stat Med. 1996;15(4):361387. doi: 10.1002/(SICI)1097-0258(19960229)15:4<361::AID-SIM168>3.0.CO;2-4 [pii].

21. R Core Team. R: A language and environment for statistical computing. R foundation for statistical computing, vienna, austria. http://www.R-project.org/. Updated 2015.

22. Moons KG, Altman DG, Reitsma JB, et al. Transparent reporting of a multivariable prediction model for individual prognosis or diagnosis (TRIPOD): Explanation and elaboration. Ann Intern Med. 2015;162(1):W1-W73. doi: 10.7326/M14-0698 [doi]. 
23. Crehange G, Roach M,3rd, Martin E, et al. Salvage reirradiation for locoregional failure after radiation therapy for prostate cancer: Who, when, where and how? Cancer Radiother. 2014;18(5-6):524534. doi: 10.1016/j.canrad.2014.07.153 [doi].

24. Aaronson DS, Yamasaki I, Gottschalk A, et al. Salvage permanent perineal radioactive-seed implantation for treating recurrence of localized prostate adenocarcinoma after external beam radiotherapy. BJU Int. 2009;104(5):600-604. doi: 10.1111/j.1464-410X.2009.08445.x [doi].

25. Beyer DC. Permanent brachytherapy as salvage treatment for recurrent prostate cancer. Urology. 1999;54(5):880-883. doi: S0090429599002411 [pii].

26. Burri RJ, Stone NN, Unger P, Stock RG. Long-term outcome and toxicity of salvage brachytherapy for local failure after initial radiotherapy for prostate cancer. Int J Radiat Oncol Biol Phys. 2010;77(5):13381344. doi: 10.1016/j.ijrobp.2009.06.061 [doi].

27. Vargas C, Swartz D, Vashi A, et al. Salvage brachytherapy for recurrent prostate cancer. Brachytherapy. 2014;13(1):53-58. doi: 10.1016/j.brachy.2013.10.012 [doi].

28. Hsu CC, Hsu H, Pickett B, et al. Feasibility of MR imaging/MR spectroscopy-planned focal partial salvage permanent prostate implant (PPI) for localized recurrence after initial PPI for prostate cancer. Int J Radiat Oncol Biol Phys. 2013;85(2):370-377. doi: 10.1016/j.ijrobp.2012.04.028;

10.1016/j.ijrobp.2012.04.028.

29. Peters M, Maenhout M, van der Voort van Zyp,J.R., et al. Focal salvage iodine-125 brachytherapy for prostate cancer recurrences after primary radiotherapy: A retrospective study regarding toxicity, biochemical outcome and quality of life. Radiother Oncol. 2014. doi: S0167-8140(14)00272-2 [pii]. 
30. Nguyen PL, Chen MH, D'Amico AV, et al. Magnetic resonance image-guided salvage brachytherapy after radiation in select men who initially presented with favorable-risk prostate cancer: A prospective phase 2 study. Cancer. 2007;110(7):1485-1492. doi: 10.1002/cncr.22934 [doi]. 